\title{
La bioéthique entre théorie et pratique, entre doctrines et contextes
}

\section{Jean Martin}

Jean Martin est ancien médecin cantonal vaudois et membre de la Commission nationale suisse d'éthique.
La European Association of Centres of Medical Ethics (EACME) organise chaque année une rencontre des personnes actives en bioéthique, notamment en milieu académique mais aussi dans des fonctions d'éthicien clinicien. Plusieurs institutions suisses en font partie. Parfaitement organisée par la Fondazione Lanza, Centre d'études avancées en éthique basé à Padoue, cette conférence a rassemblé 150 participants et avait pour thème «Multiculturalism, Religions and Bioethics».

Très impliqué dans le domaine médico-éthique et médico-légal depuis des années, je reste cependant un bioéthicien «de milice», familiarisé en emploi avec les doctrines philosophiques concernées. La réunion EACME 2007, tenue à Zurich, avait réfléchi à la professionnalisation de la bioéthique [1]. Les congrès sont de bonnes occasions d'apprécier l'évolution des idées et des pratiques et j'y participe avec intérêt, y compris à ceux de l'International Association of Bioethics [2]. Ci-dessous, des observations personnelles - et forcément sélectives - sur la réunion de Venise.

\section{La notion de responsabilité}

Elle a été discutée par F. Turoldo. Il a relevé que les œuvres classiques de morale ignoraient ce terme, qui a longtemps été confiné au domaine juridique et est présent en éthique depuis un siècle environ. Intéressant à noter, dans le sens juridique, la responsabilité est rétrospective (en cas de dommage), alors qu'en morale/éthique on la veut prospective: à l'endroit des patients mais aussi de la société et des plus vulnérables en son sein - selon Habermas et Levinas, la responsabilité des uns est proportionnelle à la vulnérabilité des autres. On sait que Max Weber distingue l'éthique de responsabilité de celle de conviction; pour J.S. Mill, l'éthique de responsabilité s'entend dans le sens de répondre pour, de prendre en charge. Aujourd'hui, on en parle dans un sens élargi, en matière de changement climatique, de ressources non-renouvelables, de démographie, de consommation.

\section{Ethique basée sur les principes et éthique basée sur les conséquences}

Débat classique: grosso modo, la première (dite aussi éthique déontologique) entend appliquer des règles vues comme immuables, sans que les conséquences de leur application jouent un rôle notable dans les déterminations, même si ces conséquences sont indé- sirables; alors que l'éthique conséquentialiste/utilitariste accorde un poids important aux effets des actions entreprises.

Les deux approches avaient leurs porte-parole à Venise. Pour ma part, tout en étant convaincu évidemment qu'il importe d'avoir des principes, je ne suis pas à l'aise avec leur application sans considération des résultats vraisemblables. Selon le prof. Turoldo, une éthique de responsabilité doit tenir compte des contextes des situations, qui parfois peuvent prévaloir sur les principes théoriques. Sur la base de sa longue expérience professionnelle, le prof. F. Abel, gynécologue-obstétricien espagnol, a plaidé pour qu'on évite de se montrer (trop) rigide au plan des principes; il a eu des mots durs pour les positions de la Curie romaine disant que les réponses qu'elle donne au début du $\mathrm{XXI}^{\mathrm{e}}$ siècle sont tout à fait anachroniques. Sur ces points, les échanges restent courtois mais l'opposition est forte entre l'intransigeance des uns et les demandes des autres pour une flexibilité tenant compte des situations concrètes. La première vertu chrétienne, a-t-il été dit, est la charité et pas la rigidité doctrinaire ou légaliste («Le sabbat et fait pour l'homme et non l'homme pour le sabbat»).

Par contraste avec une vue statique, plusieurs intervenants ont demandé que la bioéthique fasse preuve d'imagination, de créativité! Discours qui peut surprendre mais a sa pertinence.

\section{Pour une anthropologie holistique}

Une théologienne orthodoxe grecque, Angeliki Kerasidou, doctorante à Oxford, a présenté ce concept, dans l'optique humaniste d'établir un pont entre les approches chrétienne, d'une part, et laïque/profane d'autre part - notant que la valeur morale ne doit pas être vue comme une question de tout ou rien. Elle a insisté sur la complexité bio-psycho-sociale et spirituelle de l'être humain. Contre le réductionnisme, elle veut une approche systémique: «le comportement global d'un système ne peut s'expliquer par ses seules composantes, beaucoup de propriétés de la vie ne se réalisent qu'au niveau systémique». Issue de la tradition chrétienne, une telle anthropologie est aussi applicable en dehors d'elle et devrait permettre une rencontre constructive. Mais les efforts pour trouver un terrain commun d'entente sont, trop souvent, critiqués par les deux bords que l'on cherche à rapprocher! 


\section{Bioéthique au plan international}

Ce thème a été traité par Henk ten Have, directeur de la Division de l'éthique des sciences et des technologies de l'UNESCO (Paris). Il a rappelé les débuts aux Etats-Unis de la bioéthique (et la création du terme par V. R. Potter en 1970) et y voit aujourd'hui une science de la survie pour notre planète. Il s'agit d'élaborer des ponts, des solutions, à quatre niveaux: entre le présent et le futur, entre la science et les valeurs, entre la nature et la culture, enfin entre l'homme et la nature. Il a fait référence à la notion mise en évidence par l'UNESCO $\mathrm{d}^{\prime}$ «héritage commun de l'humanité». Il est urgent d'aborder pratiquement les questions de «propriétés communes de l'humanité». Cela vaut au plan culturel mais aussi au plan biologique (altérations rapides du milieu de vie, climat). En rapport avec la génétique et ses potentialités, le génome humain devrait être considéré comme un héritage commun à toute l'espèce (en vue d'éviter des utilisations inacceptables, y compris au plan commercial).

H. ten Have considère qu'un corpus éthique représente aussi un tel héritage commun, dans le sens de la Déclaration universelle des droits de l'homme de 1948 et de la Déclaration universelle sur la bioéthique et les droits de l'homme adoptée en 2005 [3]. S'agissant des interfaces entre diversité culturelle et universalisme éthique, il faut trouver les justes milieux entre homogénéisation et hétérogénéité, entre mondialisation et «localisation».

\section{«La première vertu chrétienne, a-t-il été dit, est la charité et pas la rigidité doctrinaire ou légaliste»}

Roberto Andorno, de l'Institut d'éthique biomédicale de Zurich, a parlé d'universalité des valeurs: la diversité culturelle ne doit pas mener au relativisme en éthique. Les droits de l'homme sont des garanties auxquelles chacun a droit, où qu'il ou elle vive.

Une session a été consacrée à l'idée d'une bioéthique méditerranéenne: Mme Yesim Ulman a parlé de la Turquie, soulignant que son pays, qui a une base constitutionelle laïque, entend s'approcher des pratiques occidentales, notamment dans les relations soigné-soignant (droits des patients). Toutefois, les délégués turcs ont relevé que l'attitude générale restait actuellement bien paternaliste chez eux. Salvino Leone, de Palerme a comparé les bases des traditions éthiques juive, chrétienne et musulmane. Il estime que la contribution méditerranéenne est dans le sens d'une éthique fondée sur la vertu (et l'expérience), souhaitant «intégration conviviale plutôt que confrontation dialectique». Il a mis en garde contre une «biolâtrie», la simple existence biologique ne pouvant être mise sur le même pied que la personne humaine.
Au plan général, plusieurs interventions ont insisté sur l'importance de la responsabilité éthique des pays industrialisés en ce qui concerne les défis lancés à la planète et à sa survie.

\section{A propos d'assistance au suicide et d'euthanasie}

Une session était dédiée aux sujets très actuels de l'assistance au suicide et de l'euthanasie. Le juriste R. Huxtable, de l'université de Bristol, a présenté en détail la position de la justice du Royaume-Uni vis-à-vis des personnes qui aident un proche à venir en Suisse chercher une assistance au suicide. Ceci sans qu'une conclusion claire puisse être tirée («the whole thing is as clear as mud»...). Sont en cause ici l'ordre public et l'intérêt y relatif de l'Etat mais aussi et surtout le droit à la vie privée et à agir de manière autonome (y compris celui d'aider un autre à concrétiser sa détermination). Un chiffre montre l'ambivalence des juges: depuis 2003, ce sont plus de 120 Britanniques qui sont venus terminer leurs jours à Zurich chez Dignitas, mais jusqu'ici aucun de leurs proches n'a été poursuivi en justice (alors que la loi interdit l'assistance au suicide).

Huxtable a noté un point d'importance s'agissant de l'application des lois nationales. Comme pour le suicide assisté, des questions sont posées par le tourisme de la procréation médicalement assistée: depuis plusieurs pays dont la Suisse, des couples se déplacent en Belgique ou en Espagne - ou encore en Ukraine pour une «maternité pour autrui» (mère porteuse). Il en va de même pour le tourisme en vue de transplantation, ce dernier souvent lié au trafic illégal d'organes.

J. Lemiengre et coll., de l'Université catholique de Louvain, se sont penchés sur l'impact dans trois hôpitaux flamands des prescriptions éthiques émises en application de la loi belge de 2002 autorisant l'euthanasie. Ils ont étudié l'effet de ces «documents de service» sur les attitudes et pratiques des médecins et des infirmières. Selon les premiers résultats, ce cadrage éthique a été plutôt bien ressenti par les professionnels, qui y trouvent une certaine sécurité - aussi bien ceux qui acceptent d'être partie à des euthanasies que ceux qui s'y refusent pour raisons de conscience. Une autre étude en pays flamand, par Y. Denier et coll., illustre les efforts faits par les infirmières pour apporter une réponse adéquate, au plan éthique, pratique et personnel, lors de demandes d'euthanasie.

\section{Références}

1 Martin J. Professionnaliser la bioéthique Comment? Revue médicale suisse 2008;4:484-6.

2 Martin J. La bioéthique au carrefour des avancées de la science et de cadres socio-culturels différents (Congrès de Tokyo, novembre 1998). Médecine et Hygiène. 1999;57:1837-40.

3 Martin J. Le Comité international de bioéthique (CIB) de l'UNESCO et la Déclaration universelle sur la bioéthique et les droits de l'homme. Bull Méd Suisses. 2007;88(5):190-3. 